Meta

Journal des tradlucteurs

Translators' Journal

\title{
Le Manuel du bibliothécaire documentaliste
}

\section{Louise Brunette}

Volume 25, numéro 1, mars 1980

La documentation

URI : https://id.erudit.org/iderudit/002165ar

DOI : https://doi.org/10.7202/002165ar

Aller au sommaire du numéro

Éditeur(s)

Les Presses de l'Université de Montréal

ISSN

0026-0452 (imprimé)

1492-1421 (numérique)

Découvrir la revue

Citer cet article

Brunette, L. (1980). Le Manuel du bibliothécaire documentaliste. Meta, 25(1),

84-86. https://doi.org/10.7202/002165ar d'utilisation que vous pouvez consulter en ligne.

https://apropos.erudit.org/fr/usagers/politique-dutilisation/ 


\section{Le Manuel du bibliothécaire documentaliste*}

LOUISE BRUNETTE

terminologue,

Honeywell Limitée

Publié par l'Agence de coopération culturelle et technique avec la collaboration du Conseil international de la langue française, cet ouvrage est essentiellement destiné aux non-spécialistes de la documentation. Comme son titre l'indique, il s'agit d'un ouvrage didactique : on y trouve exposées les notions fondamentales des techniques du traitement et de la communication de l'information. Parce que son caractère pédagogique le rend presque impossible à résumer, je présenterai le manuel en essayant de l'analyser objectivement malgré que cette intervention de ma part nie un principe énoncé par ses auteurs, à savoir : "L'analyse des ouvrages spécialisés est à confier à un spécialiste. 》 Dans mon approche critique, je considérerai le manuel du point de vue du responsable de l'unité documentaire d'un service de traduction.

On peut diviser le manuel en deux sections : la première partie regroupe cinq grands chapitres dans lesquels sont étudiées en détail les mailles de la chaîne documentaire ; la deuxième partie traite de l'organisation et de la gestion des ressources humaines et matérielles d'une cellule documentaire.

\section{La chaîne documentaire}

Après des considérations d'ordre général sur l'information et la documentation, les auteurs donnent une typologie des documents avant d'aborder ce qui me semble constituer le cœur du sujet, la collecte et le traitement des documents. À ce chapitre, les considérations portant sur le choix des documents sont des plus pratiques : elles guident véritablement le documentaliste sur les options qu'il doit exercer pour ce qui concerne le type de documents à acquérir, la répartition du budget, l'évaluation des sources gratuites d'approvisionnement, les sources bibliographiques à explorer et les compétences à exploiter. De plus, les auteurs commentent fort utilement divers critères de sélection de la documentation : la pertinence, la quantité, la qualité, la langue, la date et le prix des documents. L'ouvrage traite ensuite, et de façon très détaillée, de l'enregistrement et du catalogage : le texte est riche de renseignements, mais ce sont surtout les nombreux modèles de fiches donnés en exemple qui retiennent l'attention. Au

Association française des documentalistes et bibliothécaires spécialisés (ADBS) Manuel du bibliothécaire documentaliste travaillant dans les pays en développement, par M. Beauchet et autres, Paris, Presses universitaires de France (C 1977) 414 p. 
chapitre de l'analyse et de l'indexation, on s'attardera avec intérêt à la question de l'établissement du catalogue par sujets : la méthode proposée est rigoureuse et parfaitement adaptée à l'esprit comme aux besoins de services linguistiques. Vient ensuite une étude sur les langages documentaires, que les auteurs séparent en deux types : les langages à structure hiérarchique ou classifications et les langages à structure combinatoire ou lexiques. C'est à propos des premiers que sont expliquées, d'une part, les classifications encyclopédiques générales, dont la Classification décimale universelle, et, d'autre part, les classifications spécialisées, adaptées des classifications encyclopédiques pour des domaines précis d'activité. Quant à l'analyse des langages dits à structure combinatoire, elle vaut surtout par les notes qu'elle comporte sur le choix des mots clés et qui me semblent particulièrement précieuses pour le documentaliste travaillant en collaboration avec des rédacteurs, des traducteurs et des terminologues. Sous la rubrique «traitement des documents 》, on trouve égalememnt des renseignements et suggestions d'ordre pratique sur le stockage et le rangement des documents : tous ceux qui doivent constituer des dossiers documentaires liront avec profit les paragraphes qui s'y rapportent.

La première partie du manuel comporte également une étude sur l'ultime opération de la châ̂ne documentaire : la sélection. Les chapitres consacrés à la sélection mécanique ou automatique présentent à mon avis un intérêt limité puisqu'ils ne peuvent concerner que les très grands centres de documentation.

A mon point de vue, le chapitre de loin le plus intéressant est celui qui traite de l'exploitation et de la diffusion des documents. Cette partie de l'ouvrage définit, étape par étape, la démarche de recherche documentaire, depuis la prise de contact avec l'utilisateur jusqu'à la remise des documents au demandeur en passant par l'interrogation éventuelle des systèmes documentaires automatisés. Les auteurs ne se contentent pas de formuler une méthodologie : les énoncés de principe s'accompagnent d'exemples d'application des plus concrets. Encore plus précieuses sont les indications relatives aux outils à exploiter et qui invitent à l'utilisation rationnelle et maximale des diverses bibliographies d'ouvrages et de périodiques, des annuaires, des bottins de même que des catalogues et répertoires d'organismes documentaires ou autres. Conscients que la recherche documentaire est d'abord une activité de service, les auteurs s'arrêtent sur la transmission et la circulation des documents. C'est ainsi qu'ils s'intéressent aux aspects organisationnels d'un centre de documentation : mode d'accueil des usagers, constitution d'un fichier de lecteurs, formalités de consultation et de prêt. Dans le même esprit, ils renseignent les documentalistes sur les moyens à mettre en œuvre pour faire connaître la documentation aux usagers et sensibiliser ces derniers à la fonction documentaire.

\section{Organisation et gestion}

Les deux derniers chapitres de l'ouvrage s'adressent à tous ceux qui auront la responsabilité directe d'un centre documentaire existant ou à constituer. On y traite tant de l'organisation rationnelle et humaine du travail que des définitions de tâches et du recrutement du personnel. On y trouve aussi des renseignements et 
commentaires intéressants sur les divers matériels de classement, de prêt et d'exposition. Sous le titre \& Aspects psychosociaux de la communication documentaire », sont définis et analysés non seulement les rapports généraux de la cellule documentaire avec l'institution mais encore les relations personnelles entre documentalistes et usagers. Enfin, les quelques observations sur la personnalité du documentaliste guideront les intéressés dans leur recrutement. La toute dernière partie de l'ouvrage aborde l'analyse des besoins documentaires : ici encore les diverses méthodes proposées ont pour objectif de servir l'utilisateur.

En conclusion à ce résumé indicatif et critique je recommande la consultation du manuel principalement aux responsables d'unités documentaires et aux documentalistes sans formation professionnelle. Les uns comme les autres retrouveront dans les tables des matières détaillées les sujets qui les concernent. À cause même de sa destination, l'ouvrage sensibilisera les profanes aux questions documentaires et les renseignera sur l'exercice de la profession. Mais, il faut le dire, la lecture en est fastidieuse : en dépit d'une langue impeccable et d'un style clair et concis, on se sent vite étouffé par l'abondance des sujets développés. Peut-être cela tient-il à la nature, didactique, du manuel. $\grave{A}$ ce propos je veux souligner que ce livre me semble un outil pédagogique exceptionnel par la clarté des définitions, la pertinence et l'abondance des exemples, la qualité de la langue et le caractère exhaustif de son index et de ses références bibliographiques.

Je dirai en terminant que deux impressions générales se dégagent de la lecture continue du manuel : la documentation est une discipline complexe et spécialisée où l'amateurisme n'a pas sa place ; non seulement la constitution d'une unité documentaire doit-elle s'effectuer en fonction de la collectivité dans laquelle elle s'insère mais encore la cellule documentaire doit-elle faire corps avec la collectivité qu'elle doit servir. 